\section{Les ligands Delta et la niche du développement lymphoïde}

Emmanuelle Six, Isabelle André Schmutz,

Marina Cavazzana-Calvo
દ. Six, I. André Schmutz : Inserm, U768,

Paris, F-75015, France;

Université Paris-Descartes, Faculté de Médecine

René Descartes, IFR94, Paris, F-75015, France ;

M. Cavazzana-Calvo : Inserm, U768, Paris, F-75015, France ;

Université Paris Descartes, Faculté de Médecine

René Descartes, IFR94, Paris, F-75015, France ;

AP-HP, Hôpital Necker-Enfants Malades,

Département de Biothérapie, Paris, F-75015, France.

six@necker.fr tant des encoches à l'extrémité de ses ailes en raison d'une mutation dans un gène nommé alors notch (Figure 1). Les mutations dans le gène codant l'un des ligands du récepteur Notch induisent quant à elles un épaississement des veines de l'aile en forme de Delta. La voie de signalisation Notch a été conservée au cours de l'évolution et contrôle l'engagement d'un progéniteur dans une voie de différenciation donnée $[1,2]$.

عlle est essentielle à de nombreux processus au cours du développement et chez l'adulte, tels que la neurogenèse, I'hématopoïèse ou encore la différenciation des cellules épithéliales de l'intestin [3].

La transduction du signal est déclenchée par l'interaction entre un récepteur Notch et l'un de ses ligands, chacun exprimé à la surface de deux cellules voisines. II existe chez les mammifères quatre récepteurs Notch (1 à 4) et cinq ligands, Deltal, -3 et -4 et Jaggedl, -2 . Cette interaction ligand-récepteur induit deux clivages protéolytiques successifs du récepteur Notch, libérant un domaine intracellulaire actif Notch-IC, qui migre dans le noyau où il participe avec les facteurs CSL/RBPJK et Mastermind à un complexe activateur de la transcription. Ce complexe active l'expression de gènes cibles, dont les plus connus sont les facteurs de transcription de la famille bHLH : HES (hairy enhancer of split) [4] (Figure IB).

\section{Rôle}

de la signalisation Notch dans la lymphopoïèse L'implication de Notch dans des translocations chromosomiques impliquées dans des leucémies aiguës lymphoblastiques $T$ a constitué le premier pas vers la mise en évidence du rôle clé de cette molécule dans le développement lymphoïde [2].

Ce rôle a été confirmé par des expériences d'inactivation conditionnelle de Notchl à la naissance ou de surexpression de la forme active Notch-IC chez la souris. Alors que l'inactivation de Notchl conduit à un blocage complet de la différenciation lymphoïde $T$ et au développement ectopique de lymphocytes B dans le thymus [5], son expression sous forme constitutivement active induit le développement ectopique de lymphocytes $T$ dans la moelle osseuse et une inhibition simultanée du développement lymphoïde $B$ au stade pro-B [6]. Ces travaux démontrent donc que la signalisation via le récepteur Notchl joue un rôle fondamental dans l'induction de la différenciation lymphoïde T via, entre autres, l'activation du gène $p T \alpha$, et l'inhibition du développement lymphoïde B par la répression du facteur Pax 5 , indispensable à l'initiation du développement lymphoïde B [7] (Figure 1B).

\section{Les ligands Delta}

dans la différenciation lymphoïde $T$ : rôle du microenvironnement

Si le rôle du récepteur Notch est bien caractérisé, en revanche la question du ou des ligands responsable(s) du contrôle de la différenciation lymphoïde est restée longtemps en suspens. L'activation de la signalisation Notch dans le compartiment hématopoïétique met en jeu une communication intercellulaire entre les cellules souches hématopoïétiques (CSH) et les cellules stromales qui constituent leur microenvironnement. L'analyse comparative du profil d'expression des ligands de Notch dans la moelle osseuse et le thymus murins a révélé que les ligands Deltal et Delta4 ont une expression restreinte au microenvironnement thymique et sont particulièrement concentrés dans la région corticale et à la jonction corticomédullaire où arrivent via la circulation les progéniteurs lymphoïdes produits dans la moelle osseuse

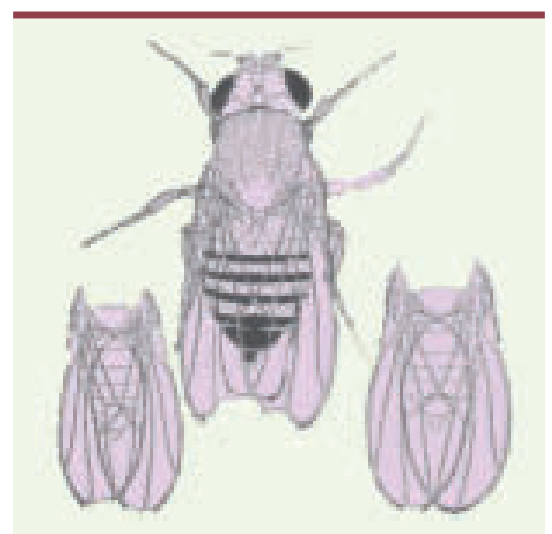

Figure 1. Souche de drosophile mutante (Thomas Morgan, 1917). 
et où se produisent les premières étapes du développement thymique [8] (Figure 2A). Les ligands Jaggedl/2 sont quant à eux exprimés à la fois dans la moelle osseuse et dans le thymus. Le rôle primordial des ligands de la famille Delta dans l'induction de la différenciation lymphoïde $T$ a été confirmé par des expériences menées chez la souris nude naturellement dépourvue de thymus. La surexpression ectopique du ligand Deltal ou Delta4 dans les CSH de

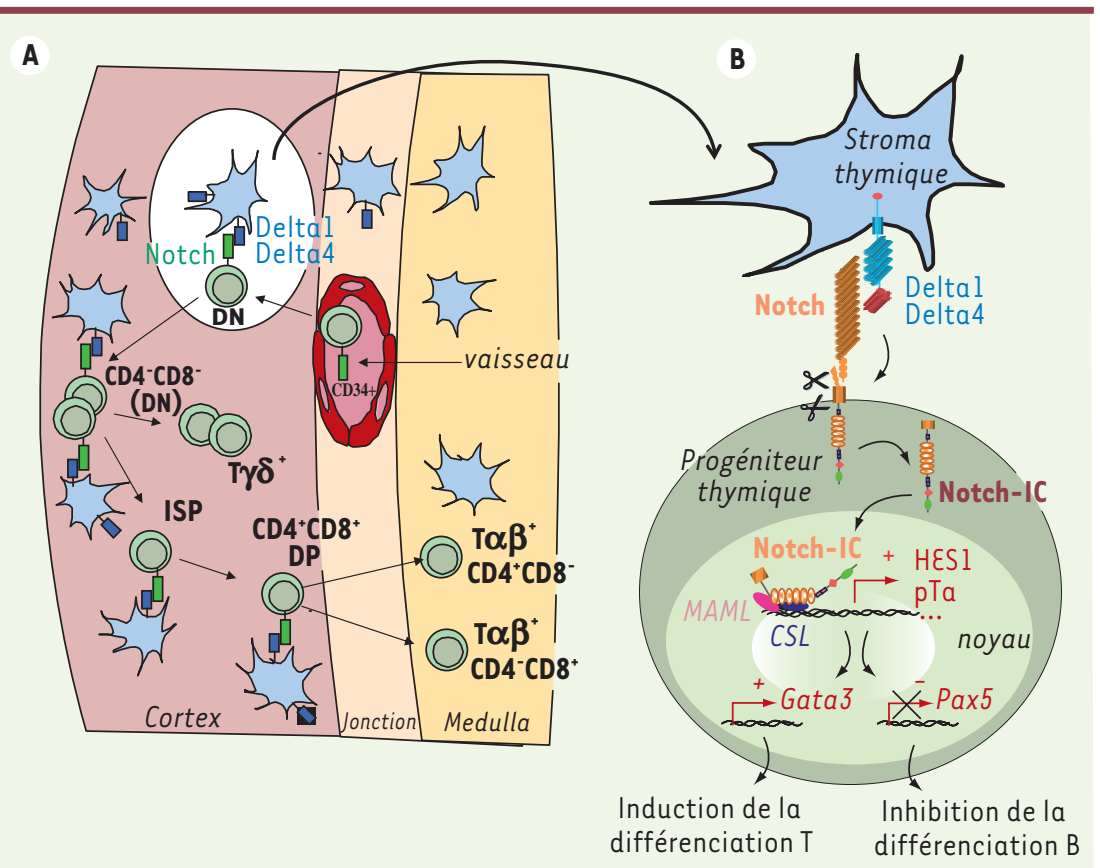

Figure 2. Architecture thymique chez l'adulte et transduction du signal Delta/Notch. A. Le thymus est colonisé par un progéniteur pluripotent, exprimant le marqueur $C D 34^{+}$, au niveau de la jonction cortico-médullaire. L'interaction de ce progéniteur avec les cellules épithéliales corticales environnantes qui expriment le ligand Deltal et/ou Delta4 induit l'engagement dans le lignage lymphoïde T (voir agrandissement en $\boldsymbol{B}$ ). Ce progéniteur passe alors par plusieurs stades successifs de différenciation au sein du cortex, régulés par la signalisation Delta/Notch: cellules double-négatives CD4CD8- (DN), immatures simple-positives CD4 $4^{+}$(ISP), doubles positives CD4 ${ }^{+} C D 8^{+}$(DP). Les lymphocytes T exprimant le TCR $\gamma \delta$ sont également engendrés au sein du cortex et dérivent des cellules DN. Au cours de l'étape DP, les précurseurs thymiques exprimant le TCR $\alpha \beta$ à leur surface reconnaissent I'un des deux complexes majeurs d'histocompatibilité $\mathrm{CMH}$, et se différencient en précurseurs simple-positifs $\mathrm{CD} 4^{+}$(SP4) ou CD8 $8^{+}$(SP8) qui vont achever leur maturation dans la région médullaire. B. À la suite de l'interaction avec l'un de ses ligands Delta, le récepteur Notch subit deux clivages protéolytiques qui libèrent le domaine intracellulaire du récepteur Notch-IC dans le cytoplasme. Notch-IC migre alors dans le noyau où il participe à un complexe activateur de la transcription avec les facteurs CSL et Mastermind (MAML), qui active les gènes cibles tels que HESl ou encore la chaîne $\alpha$ du pré-TCR, pTa. S'ensuit une cascade d'événements transcriptionnels, entre autres l'activation du gène Gata 3 favorisant l'engagement dans le lignage lymphoïde T et la répression du gène Pax5, inhibant ainsi l'engagement dans le lignage lymphoïde B. de réguler la motilité cellulaire [10]. Les propriétés intrinsèques des ligands Delta pourraient ainsi favoriser les connexions entre les cellules stromales et participer à la constitution d'une niche thymique privilégiée permettant l'induction de la différenciation $\mathrm{T}$.

\section{Différenciation lymphoïde $\mathrm{T}$ in vitro}

La mise en évidence du rôle essentiel des ligands Delta dans le développement lymphoïde $T$ a eu une application majeure: le développement d'un système de différenciation lymphocytaire $T$ in vitro constituant une alternative très intéressante au système de culture organotypique de thymus fœtal jusqu'alors utilisé, difficile à manipuler et peu efficace. L'équipe de J.C. Zúñiga-Pflücker a ainsi démontré que la surexpression de Deltal dans la lignée stromale murine de moelle osseuse OP9 induit deux effets notables: la lignée OP9-mDeltal perd sa capacité de permettre la lymphopoïèse $B$, et acquiert celle d'induire le développement de lymphocytes $T$ immatures double-positifs $\mathrm{CD}^{+} \mathrm{CD} 8^{+}$ainsi que de lymphocytes $T$ matures simple-positif CD8 ${ }^{+}$SP [11] (Figure 3).

Chez l'homme, les étapes précoces du développement $T$ sont mal connues, du fait de l'absence de modèles d'études efficaces. Une avancée cruciale a donc été franchie dans l'étude de la lymphopoïèse humaine grâce à l'adaptation du système de culture OP9-Deltal à la différenciation $T$ à partir de progéniteurs humains $[12,13]$ (Figure 3 ). Notre équipe a développé ce système biologique en construisant une lignée $O P 9$ exprimant le ligand Deltal humain (OP9-hDeltal). Nous avons ainsi pu suivre les étapes de la lymphopoïèse en partant de différentes populations cellulaires isolées du sang de cordon ou de moelle osseuse humaine. Après quatre semaines de coculture avec la lignée OP9-hDeltal, les CSH se différencient de manière reproductible en lymphocytes T CD $4^{+} C D 8^{+}$DP et en lymphocytes TTCR $\gamma \delta^{+}$. Une semaine supplémentaire est nécessaire pour observer l'apparition de quelques lymphocytes $T$ matures expri- 
mant le récepteur T à l'antigène TCR $\alpha \beta^{+}$. Le biais de développement vers la lignée $\mathrm{T} T \mathrm{TCR} \gamma \delta^{+}$au détriment de la lignée $\mathrm{T}$ TCR $\alpha \beta^{+}$, observé de manière constante dans ce système de culture, pourrait s'ex- pliquer par l'absence de CMH (complexe majeur d'histocompatibilité) humains dans ce système, ou par l'implication de Notch à d'autres stades de différenciation $T$ dans la régulation des réarrangements

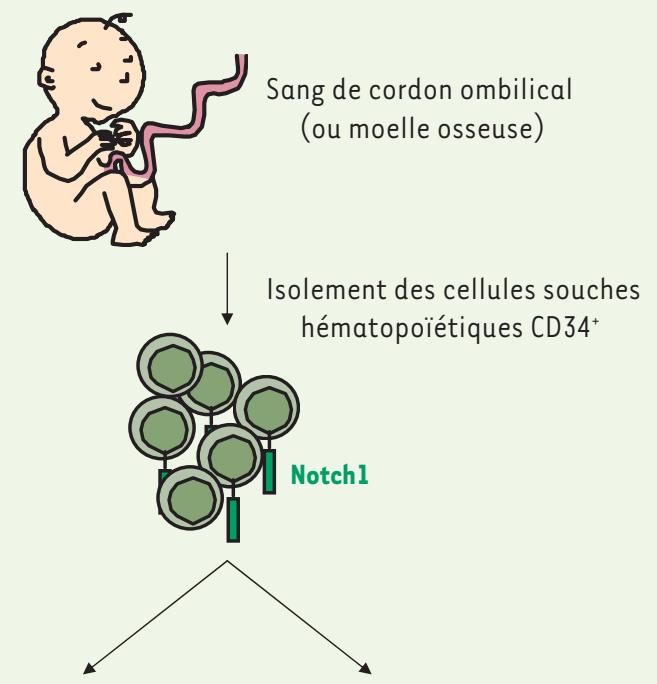

A Stroma OP9

Expression Jagged 1/2

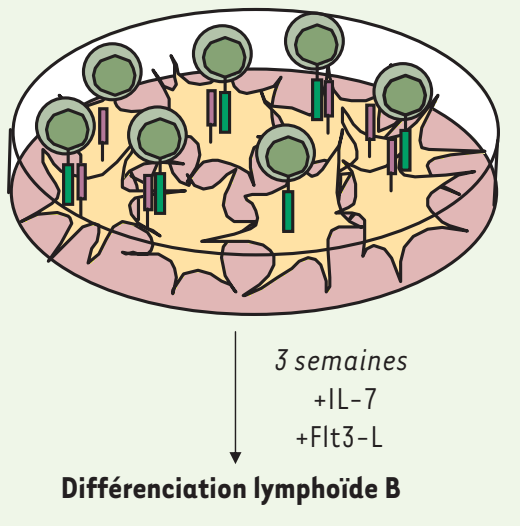

B Stroma OP9-hDeltal

Expression Jaggedl/2 + hDeltal

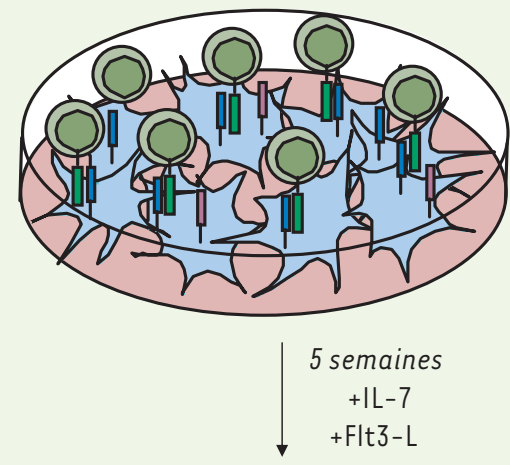

Différenciation lymphoïde $T$
Figure 3. Modèles de différenciation lymphoïde humaine in vitro. Les cellules souches hématopoïetiques (CSH) humaines portant le marqueur de surface $\mathrm{CD} 34^{+}$et dont une partie exprime le récepteur Notchl, sont isolées à partir de sang de cordon ombilical ou de la moelle osseuse adulte. Les CSH sont alors cultivées pendant plusieurs semaines en présence de cytokines (IL-7 et ligand de Flt3 [Flt3-L]) et d'un support de cellules stromales qui miment soit le microenvironnement médullaire de la moelle osseuse (OP9), soit le microenvironnement thymique (OP9-hDeltal). A. Le stroma OP9 est une lignée

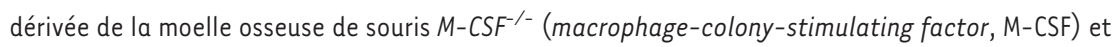
qui exprime de manière endogène les ligands Jaggedl et Jagged2. Ce stroma induit au bout de trois semaines une différenciation lymphoïde B des CSH et reproduit ainsi la différenciation observée dans la moelle osseuse. $B$. Le stroma OP9-hDeltal est dérivé de la lignée OP9 décrite précédemment en y surexprimant le ligand Deltal humain de manière ectopique. L'expression du ligand Deltal induit une inhibition complète du développement $B$ et conduit après environ 5 semaines à une différenciation lymphoïde T. Ce stroma permet de reproduire in vitro les étapes précoces de la différenciation thymique jusqu'au stade DP et lymphocytes $T \gamma \delta$. NB : Des résultats analogues sont obtenus à partir de CSH murines mises en culture avec la lignée OP9 ou OP9-mDeltal. des gènes codant la chaîne $\beta$ du TCR, le contrôle des décisions $\alpha \beta$ versus $\gamma \delta$ ou encore CD4 versus CD8 [14].

Au-delà de cette observation, l'intérêt majeur de ce système de différenciation in vitro est de permettre des analyses fines des premières étapes de différenciation T, l'identification des progéniteurs hématopoïétiques investis d'un potentiel T au sein d'une population hétérogène, l'identification de l'impact de diverses cytokines et facteurs de croissance sur la différenciation T. II a été ainsi démontré que le ligand Deltal purifié permettait à lui seul l'engagement des CSH dans la voie de différenciation $T$, avec un effet dose important [15], mais que l'obtention de cellules $T$ matures nécessitait la présence d'un stroma (OP9 dans ce système). Afin de modéliser de manière plus fidèle le microenvironnement thymique, des systèmes dérivés devront être explorés afin de définir la combinaison idéale de cytokines, ainsi que les molécules impliquées dans les étapes de sélection positive et négative, telles que les composants du CMH ou AIRE ${ }^{1}$ qu'il faudrait ajouter pour compléter les étapes terminales de la différenciation. Ainsi, si nos avancées dans la définition des acteurs des niches hématopoïétiques tels que les ligands Delta nous ont d'ores et déjà permis d'engendrer des lymphocytes $T$ humain in vitro, ce n'est qu'une première étape ouvrant de nombreuses perspectives dans l'étude des pathologies du système immunitaire et leur correction par thérapie génique ou cellulaire. La première de ces perspectives pourrait concerner le problème majeur de la reconstitution immunitaire après greffe de cellules souches hématopoïétiques partiellement incompatibles, dont la résolution repose sur l'identification de progéniteurs lymphoïdes capables de rapidement se différencier en lymphocytes T. $\diamond$

Notch ligands Delta and

lymphoid development niches

${ }^{1}$ AIRE (autoimmune regulator) est un facteur de transcription exprimé dans les cellules épithéliales de la médullaire thymique, et dont les mutations du gène sont responsables du syndrome auto-immun polyendocrine de type l (APS1) ou autoimmune-polyendocrinopathy-candidiasis-ectodermal dystrophy (APECED). 


\section{RÉFÉRENCES}

1. Brou C, Logeat F. Endocytose et voie de signalisation Notch. Med Sci (Paris) 2006 ; $22: 685-8$.

2. Kastner $P$, Chan $S$. La voie Notch au centre du mécanisme de leucémogenèse dans un modèle murin de leucémies T. Med Sci (Paris) 2006 ; 22 :708-10.

3. Robine $S$, Fre $S$, Huyghe $M$, et al. Notch, un gène clé de l'équilibre entre prolifération et différenciation des cellules épithéliales de l'intestin. Med Sci (Paris) $2005 ; 21: 780-2$.

4. Schweisguth F. Notch signaling activity. Curr Biol 2004 ; 14 : R129-38.

5. Radtke F, Wilson A, Stark G, et al. Deficient T cell fate specification in mice with an induced inactivation of Notch 1. Immunity 1999; $10: 547-58$

6. Pui JC, Allman D, Xu L, et al. Notchl expression in early lymphopoiesis influences $B$ versus $T$ lineage determination. Immunity 1999; 11 : 299-308.
7. Hoflinger S, Kesavan K, Fuxa M, et al. Analysis of notchl function by in vitro $T$ cell differentiation of pax5 mutant lymphoid progenitors. J Immunol 2004 ; $173: 3935-44$.

8. Schmitt TM, Ciofani M, Petrie HT, et al. Maintenance of $T$ cell specification and differentiation requires recurrent notch receptor-ligand interactions. J Exp Med $2004 ; 200: 469-79$.

9. De La Coste A, Six $\varepsilon$, Fazilleau N, et al. In vivo and in absence of a thymus, the enforced expression of the Notch ligands delta- 1 or delta-4 promotes T cell development with specific unique effects. J Immunol $2005 ; 174: 2730-7$.

10. Six EM, Ndiaye D, Sauer G, et al. The notch ligand Deltal recruits DIgl at cell-cell contacts and regulates cell migration. J Biol Chem 2004 ; 279 : 55818-26.

11. Schmitt TM, Zuniga-Pflucker JC. Induction of T cell development from hematopoietic progenitor cells by delta-like-1 in vitro. Immunity $2002 ; 17: 749-56$.

\section{NOUVELLE}

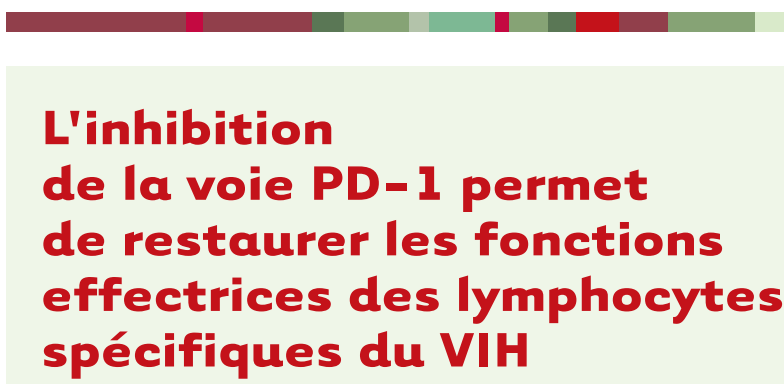

Lydie Trautmann, Nicolas Chomont, Rafick-Pierre Sékaly

$>$ Lors d'une infection virale chronique, le système immunitaire est incapable d'éliminer totalement le virus et celui-ci peut persister longtemps dans certains compartiments cellulaires ou anatomiques. Dans la plupart des cas, le système immunitaire met en place une réponse mémoire efficace permettant de contenir les épisodes de réactivation du virus. En revanche, l'infection par le VIH (virus de l'immunodéficience humaine) s'accompagne de la perte de la capacité des lymphocytes $T$ mémoires à juguler la réplication virale, malheureusement les causes de cet état dysfonctionnel ne sont pas encore bien comprises [1]. De nombreuses études ont mis en évidence l'absence de fonctions effectrices des cellules T CD8 spécifiques du VIH et leur incapacité à proliférer [2-5]. Plusieurs stratégies ont été déployées pour essayer de restaurer ces fonctions, par exemple: la trithérapie, l'ajout de cellules compétentes CD4 spécifiques du VIH ou encore de cytokines. Bien que ces dernières aient permis d'améliorer la prolifération des cellules CD8 spécifiques du $\mathrm{VIH}$, la restauration des fonctions effectrices n'a jamais été observée jusqu'à présent.

La régulation des réponses $T$ aux infections virales résulte d'un équilibre délicat entre leurs fonctions effectrices nécessaires pour éliminer l'agent pathogène et leur potentiel à causer des dommages aux tissus. Ainsi, les molécules régulant le système immunitaire ont une importance capitale dans le maintien de l'équilibre entre une réponse efficace et le développement de pathologies autoimmunes. Parmi ces molécules régulatrices, la molécule PD-1 (Programmed Death-1, une protéine exprimée à la surface de certains lymphocytes) permet d'éviter une activation excessive de la réponse immunitaire [6]. Plusieurs équipes ont montré que l'entrée en jeu de PD-1 avec son ligand PD-Ll atténue la voie de signalisation du récepteur des cellules T, responsable de la reconnaissance spécifique des antigènes viraux et de
12. La Motte-Mohs RN, Herer $\varepsilon$, Zuniga-Pflucker JC. Induction of T-cell development from human cord blood hematopoietic stem cells by Delta-like 1 in vitro. Blood $2005 ; 105$ : 1431-9.

13. De Smedt M, Hoebeke I, Plum J. Human bone marrow CD34+ progenitor cells mature to T cells on OP9-DL1 stromal cell line without thymus microenvironment. Blood Cells Mol Dis 2004 ; 33 : 227-32.

14. Maillard I, Fang T, Pear WS. Regulation of lymphoid development, differentiation, and function by the Notch pathway. Annu Rev Immunol 2005 ; 23 : 945-74

15. Delaney C, Varnum-Finney B, Aoyama K, et al. Dosedependent effects of the Notch ligand Deltal on ex vivo differentiation and in vivo marrow repopulating ability of cord blood cells. Blood 2005 ; 106 : 2693-9.

Laboratoire d'Immunologie,

Centre de recherche du Centre Hospitalier de I'Université de Montréal-Saint-Luc, Inserm U743, 264, boulevard René-Lévesque Est, Montréal (Québec) H2X IPl Canada. lydie.trautmann@umontreal.ca

l'initiation de la réponse des lymphocytes T, et induit ainsi l'inhibition de la prolifération et de la production d'IL-2 [7]. Récemment, l'équipe du Dr Rafi Ahmed a mis en évidence le rôle de PD-1 dans le dysfonctionnement des cellules CD8 au cours de l'infection chronique par le virus de la chorioméningite lymphocytaire (LCMV) chez la souris. L'administration d'anticorps empêchant la liaison de PD-1 avec son ligand permettait de restaurer la fonction des cellules CD8 spécifiques du virus et de réduire la charge virale des souris infectées [8]. Ces résultats nous ont incités à étudier le rôle de PD-1 dans l'infection par le VIH.

Dans cette étude, nous avons étudié l'expression de la molécule PD-1 sur des cellules CD8 spécifiques du VIH, du CMV (cytomégalovirus) et de l'EBV (virus d'Epstein-Barr) au sein des mêmes groupes de patients [9]. Nous avons montré que l'expression de PD-1 était plus importante sur les cellules CD8 VIH-spécifiques que sur les cellules CMV- et 